\title{
Influence of Lifestyle Aspects on the Association of Body Size and Shape with All-Cause Mortality in Middle-Aged Men and Women
}

\author{
Janne Bigaarda,b,c Jane Christensen ${ }^{a} \quad$ Anne Tjønneland ${ }^{a} \quad$ Birthe Lykke Thomsen $^{a}$ \\ Kim Overvad ${ }^{b}$ Thorkild I.A. Sørensen ${ }^{c}$ \\ a Institute of Cancer Epidemiology, The Danish Cancer Society, Copenhagen, \\ ${ }^{b}$ Department of Clinical Epidemiology, Aalborg Hospital, Aarhus University Hospital, Aalborg, \\ ${ }^{c}$ Institute of Preventive Medicine, Copenhagen University Hospital, Center for Health and Society, Denmark
}

\section{Keywords}

Lifestyle - Waist circumference - Body mass index . Hip circumference - All-cause mortality

\section{Summary}

Objective: Waist circumference, BMI and hip circumference are differentially associated with mortality. We investigated the potential influence of selected lifestyle aspects such as smoking, alcohol intake, sports activity and education. Method: The Danish prospective study 'Diet, Cancer and Health' recruited 27,179 men and 29,875 women from 1993 to 1997 . Cox regression models were used to estimate mortality rate ratios. Results: Adjustment for smoking habits attenuated the associations between mortality and the three body size measurements in both sexes. Adjustment for sport activity and, to a lesser extent, alcohol intake weakened the associations further for the men, whereas alcohol intake slightly weakened associations for the women. In the fully adjusted models, mortality increased highly significantly with higher waist circumference and lower hip circumference, and decreased highly significantly with higher BMI for BMI below $25 \mathrm{~kg} / \mathrm{m}^{2}$. This pattern was seen for all levels of the selected lifestyle factor. Conclusion: A large waist circumference remained a strong risk indicator for mortality, and a large hip circumference appeared to be protective when smoking habits, alcohol intake and sport activity were accounted for. BMI below $25 \mathrm{~kg} / \mathrm{m}^{2}$ remained a risk factor, but not above $25 \mathrm{~kg} / \mathrm{m}^{2}$ once waist circumference was adjusted for.

\section{Introduction}

In previous prospective studies we found an inverse association between BMI (= weight $/ \mathrm{height}^{2} ; \mathrm{kg} / \mathrm{m}^{2}$ ) and mortality when comparing individuals with similar waist circumferences and a direct association between waist circumference and mortality when comparing individuals of similar BMI [1]. We have also found an inverse association with mortality when comparing individuals of similar waist circumference [2]. In these studies we adjusted for smoking, but not for other lifestyle aspects, such as alcohol intake, physical activity and social position, and we did not present separate risk estimates for the different aspects of smoking habits $[1,2]$. In view of the intimate relationship between these lifestyle aspects and both obesity and mortality the question prevails whether they have confounding influences on the associations while assuming that they are not mediators of the associations (intermediates between the anthropometric measurements and the outcome) [3-5]. However, it is equally important to determine whether lifestyle aspects have modifying influences on the associations.

The aim of this study was therefore to evaluate whether the selected lifestyle aspects influenced the previously shown association between mortality and the three body size measurements: waist circumference, hip circumference and BMI. We considered waist circumference together with BMI and waist circumference together with hip circumference. We investigated the effect of inclusion of the selected lifestyle aspects as potential confounders and furthermore studied potential effect modification by the selected lifestyle factors.

\section{KARGER}

Fax +497614520714

Information@Karger.de

www.karger.com (c) 2010 S. Karger GmbH, Freiburg

Accessible online at:

www.karger.com/ofa 


\section{Material and Methods}

Between December 1993 and May 1997, a total of 160,725 persons aged 50-64 years were invited to participate in the Danish prospective study 'Diet, Cancer and Health'. Potential participants were all inhabitants of the greater Copenhagen or Aarhus areas, born in Denmark, and with no record of cancer registered in the Danish Cancer Register at the time of invitation. In all, 27,178 men (33.6\% of total number eligible) and 29,875 women (37.5\% of total number eligible) participated, comprising $7 \%$ of the entire Danish population in this age group. The study was conducted in accordance with Helsinki Declaration II and approved by the Ethical Committees on Human Studies in Copenhagen and Aarhus municipalities [6].

\section{Body Size Measurements}

All body size measurements were collected at two study clinics situated in Aarhus and Copenhagen. Trained laboratory technicians obtained body size measurements. Height was measured with the participants standing without shoes and was recorded to the nearest $0.5 \mathrm{~cm}$. Weight was measured by a digital scale, with the participants wearing light clothing or underwear, and was recorded to the nearest $100 \mathrm{~g}$. Waist circumference was measured at the narrowest part between the lower rib and the iliac crest (the natural waist) or, in case of an indeterminable waist narrowing, halfway between the lower rib and the iliac crest, and was recorded to the nearest $0.5 \mathrm{~cm}$. Hip circumference was measured over the widest part of the buttocks, and was recorded to the nearest $0.5 \mathrm{~cm}$.

\section{Lifestyle Aspects}

Smoking variables were defined from a lifestyle questionnaire completed at the study clinics at enrolment. Questionnaires were scanned immediately, and missing answers were filled out during a personal interview at the clinic. We used information about smoking status (current, recent, past and never), current tobacco consumption (g/day), time since smoking cessation (years), and smoking duration (years). Recent smoking, defined as smoking cessation within the last year, was examined as a separate category for smoking status, because we considered these participants at high risk for resuming smoking. Current tobacco consumption was calculated in $\mathrm{g} /$ day using conversion factors of 1 (cigarettes), 4.5 (cigars), 3 (cheroots), and 3 (pipe). Alcohol intake was considered as abstainers (reporting no alcohol intake in both the dietary and lifestyle questionnaire), occasional drinkers (those reporting no alcohol intake in one questionnaire (dietary or lifestyle questionnaire) but not the other) and the remaining group. For this remaining group alcohol intake was considered as average daily alcohol intake in g/day. Information about physical activity was obtained as number of hours per week spent on six different types of physical activities in leisure-time. Education was considered as years of schooling in three categories; below 8 years of schooling, between 8 and 10 years, and above 10 years.

\section{Exclusions}

Some participants were diagnosed with a cancer close to their visit in the study center. Due to the delay in registration of the cancer diagnosis in the Danish Cancer Registry invitations were sent out anyway. When these participants were informed about their cancer diagnosis, they were not rejected at the study clinics. Instead they were excluded from the study population before analyses (569 out of a total of 57,053 (1.0\%)). We further excluded 56 participants who had missing values of waist or hip circumferences, weight or height measurements. 19 participants were excluded because of implausible values of body size measurements in the database. For 569 participants (1.0\%) information about smoking habits was missing (smoking status $(\mathrm{n}=70)$; duration $(\mathrm{n}=197)$; time since cessation $(\mathrm{n}=279)$; and current consumption $(\mathrm{n}=8))$, and 15 had extreme values ( 9 had tobacco consumption $>100 \mathrm{~g} / \mathrm{day}, 3 \mathrm{had}$ negative smoking duration, 3 never smokers indicated smoking start). We missed data on alcohol intake for 49 participants, on physical activity for 1,508 participants and on education for 26 participants. A total of 54,257 participants (95\%) were eligible for analyses: 25,901 men and 28,356 women.

\section{Endpoint and Follow- $U p$}

All-cause mortality was the endpoint in the study. Complete follow-up of emigration and vital status until December 31, 2005 was obtained through record-linkage to The Civil Registration System, using the unique personal identification number assigned to each inhabitant of Denmark.

\section{Statistical Analyses}

We estimated the associations between the body size measurements, waist circumference, hip circumference and BMI, and the sex-specific allcause mortality using Cox proportional hazards models. This produced estimates of mortality rate ratios (MRR) and corresponding confidence limits $(95 \%)$. Age was used as the time axis to ensure that the estimation procedure was based on comparisons of individuals at the same age, which optimizes control for potential confounding from differences in age [7]. The analyses were corrected for delayed entry so that individuals were only considered at risk from the age at entry into the study until death, emigration, time of disappearance or December 31, 2005 whichever came first. The time since baseline examination was modeled as a time-dependent linear spline, that is, a piecewise linear function connected at joint points (knots) [8] placed at 1 year, 2 years and 3 years, allowing the hazard to change with time under study. Separate analyses were performed for men and women due to possible differences in effects of body size and body composition.

The measured waist and hip circumferences and BMI were log-transformed and initially modeled using linear splines. Linear splines consider the variation in mortality risk within intervals (delimited by the knots) as well as between intervals, estimating linear effects within each interval and allowing the slope to change at the knots [8]. Using linear splines we gained the advantages of a continuous modeling of the effect of a continuous exposure variable without having to assume a linear association across the whole spectrum of the exposure variable. The knots for the log-transformed waist and hip circumferences were placed at the quartiles among the deceased participants, whereas the knots for the log-transformed BMI were placed at the category limits specified in the WHO BMI criteria, [9] that is, at $18.5,25$, and $30 \mathrm{~kg} / \mathrm{m}^{2}$. For the log-transformed waist and hip circumferences, the associations were linear over the whole range of observed values. Therefore, the effects of these two log-transformed measurements were simplified to straight lines in the models presented in this paper.

Body size measurements were modeled in two different ways: In one model, the log-transformed waist circumference and BMI were considered mutually adjusted. In the other model, the log-transformed waist and hip circumferences were considered mutually adjusted. The inclusion of log-transformed waist and hip circumferences as linear variables, mutually adjusted, is a more flexible model than the usual waist-to-hip ratio, and this model makes it possible to test whether or not the waist-to-hip ratio is an appropriate combination of the two measurements. If the waist-to-hip ratio is appropriate, then the rate ratio corresponding to a $10 \%$ higher waist circumference should equal the rate ratio corresponding to a $10 \%$ lower hip circumference.

We evaluated the confounding potential of each of the lifestyle aspects (smoking habits, alcohol intake, sport activity and education) on the associations between the body size measurements and all-cause mortality for each of the two models mentioned above. The association between mortality and the body size measurements estimated without any adjustment for lifestyle was compared to the associations adjusted for each of the lifestyle aspects, one at a time. Likewise, the association between mortality and the body size measurements estimated in a full model adjusted for all the lifestyle aspects was compared to the estimated associations when each lifestyle aspect was excluded one at a time from the full model. 
Table 1. Characteristics of selected lifestyle aspects according to waist circumference quartiles among deceased men. Crude and adjusted mortality rate ratio (MRR) for each lifestyle aspect.

\begin{tabular}{|c|c|c|c|c|c|c|c|c|}
\hline & \multicolumn{4}{|c|}{ Waist circumference, $\mathrm{cm}$} & \multicolumn{2}{|c|}{$\operatorname{MRR}(95 \% \mathrm{CI})^{*}$} & \multicolumn{2}{|c|}{$\operatorname{MRR}(95 \% \mathrm{CI})^{\dagger}$} \\
\hline & under 89 & $89-94.5$ & $95-101.5$ & over 102 & & & & \\
\hline \multicolumn{9}{|l|}{$\overline{M e n}$} \\
\hline Number of deaths & 586 & 553 & 550 & 534 & & & & \\
\hline Number in cohort & 7,625 & 7,863 & 6,420 & 3,966 & & & & \\
\hline \multicolumn{9}{|l|}{ Lifestyle aspects } \\
\hline Never smokers, \% $(\mathrm{N})$ & $28(2,145)$ & $27(2,099)$ & $24(1,557)$ & $24(948)$ & 1.00 & - & 1.00 & - \\
\hline Current smokers, \% (N) & $45(3,396)$ & $38(3,017)$ & $37(2,374)$ & $37(1,475)$ & $2.67 \ddagger$ & $(2.32-3.08)$ & $2.32 \ddagger$ & $(2.01-2.68)$ \\
\hline Recent smokers, \% (N) & $1(102)$ & $1(111)$ & $2(101)$ & $2(63)$ & $1.55^{\S}$ & $(1.02-2.36)$ & $1.39^{\S}$ & $(0.91-2.12)$ \\
\hline Past smokers, \% (N) & $26(2,009)$ & $34(2,636)$ & $37(2,388)$ & $37(1,480)$ & $1.37 * *$ & $(1.17-1.59)$ & $1.27 * *$ & $(1.09-1.48)$ \\
\hline $\begin{array}{l}\text { Current tobacco consumption }{ }^{\dagger \dagger} \text {, median } \\
\quad \text { (range) }\end{array}$ & $20(5-40)$ & $20(4-40)$ & $20(4-40)$ & $20(5-44)$ & 1.29 & $(1.21-1.38)$ & 1.20 & $(1.12-1.27)$ \\
\hline Time since cessation ${ }^{\ddagger}$, median (range) & $18(2-35)$ & $16(2-34)$ & $14(1-34)$ & $14(1-33)$ & 0.96 & $(0.89-1.03)$ & 0.94 & $(0.88-1.01)$ \\
\hline Smoking duration $^{\S \S}$, median (range) & $34(7-47)$ & $33(7-47)$ & $33(8-47)$ & $33(8-48)$ & 1.24 & $(1.11-1.38)$ & 1.17 & $(1.06-1.30)$ \\
\hline Alcohol abstainers, \% (N) & $2(146)$ & $1(109)$ & $1(83)$ & $2(71)$ & $3.43^{* * *}$ & $(2.75-4.28)$ & $2.68 * * *$ & $(2.15-3.36)$ \\
\hline Occasional drinkers, \% (N) & $1(91)$ & $1(72)$ & $1(70)$ & $1(48)$ & $2.48 * * *$ & $(1.89-3.27)$ & $2.02 * * *$ & $(1.53-2.66)$ \\
\hline Alcohol $^{\dagger \dagger \dagger}$, median (range) & $19(2-71)$ & $20(3-75)$ & $20(3-82)$ & $20(2-90)$ & 1.10 & $(1.09-1.12)$ & 1.07 & $(1.06-1.09)$ \\
\hline No sports activity, \% (N) & $45(3,448)$ & $47(3,678)$ & $55(3,507)$ & $66(2,634)$ & $1.78^{\ddagger \ddagger}$ & $(1.60-1.98)$ & $1.40^{\ddagger \neq ⿱ 亠 䒑 十}$ & $(1.26-1.57)$ \\
\hline Duration of sports activity, median (range) & $2(0.5-7.5)$ & $2(0.5-7.5)$ & $2(0.5-7.5)$ & $2(0.5-7.5)$ & $1.01^{\S \S}$ & $(0.98-1.04)$ & $1.01^{\S \S}$ & $(0.98-1.04)$ \\
\hline$<8$ years of school, $\%(\mathrm{~N})$ & $29(2,221)$ & $32(2,550)$ & $37(2,404)$ & $44(1,761)$ & 1.00 & - & 1.00 & - \\
\hline $8-10$ years of school, $\%(\mathrm{~N})$ & $43(3,249)$ & $43(3,343)$ & $41(2,631)$ & $40(1,569)$ & 0.80 & $(0.73-0.87)$ & 0.87 & $(0.80-0.96)$ \\
\hline \multirow[t]{3}{*}{$>10$ years of school, $\%(\mathrm{~N})$} & $29(2,182)$ & $25(1,970)$ & $22(1,385)$ & $16(636)$ & 0.66 & $(0.59-0.74)$ & 0.80 & $(0.71-0.90)$ \\
\hline & \multicolumn{4}{|c|}{ Waist circumference, $\mathrm{cm}$} & \multirow{2}{*}{\multicolumn{2}{|c|}{$\operatorname{MRR}(95 \% \mathrm{CI}) *$}} & \multirow{2}{*}{\multicolumn{2}{|c|}{$\operatorname{MRR}(95 \% \mathrm{CI})^{\dagger}$}} \\
\hline & under 74 & $74-79.5$ & $80-87.5$ & over 88 & & & & \\
\hline \multicolumn{9}{|l|}{ Women } \\
\hline Number of deaths & 367 & 324 & 366 & 324 & & & & \\
\hline Number in cohort & 7,641 & 7,909 & 7,569 & 5,239 & & & & \\
\hline \multicolumn{9}{|l|}{ Lifestyle aspects } \\
\hline Never smokers, \% (N) & $44(3,364)$ & $44(3,484)$ & $44(3,299)$ & $45(2,369)$ & 1.00 & - & 1.00 & - \\
\hline Current smokers, \% (N) & $35(2,709)$ & $32(2,566)$ & $32(2,405)$ & $30(1,566)$ & $3.38^{\ddagger}$ & $(2.90-3.93)$ & $2.94^{\ddagger}$ & $(2.52-3.43)$ \\
\hline Recent smokers, \% (N) & $1(76)$ & $1(86)$ & $1(91)$ & $1(61)$ & $1.22^{\S}$ & $(0.65-2.29)$ & $1.18^{\S}$ & $(0.63-2.20)$ \\
\hline Past smokers, \% (N) & $20(1,492)$ & $22(1,771)$ & $23(1,774)$ & $24(1,243)$ & $1.65^{* *}$ & $(1.38-1.98)$ & $1.63^{* *}$ & $(1.36-1.96)$ \\
\hline $\begin{array}{l}\text { Current tobacco consumption }{ }^{\dagger \dagger} \text {, median } \\
\quad \text { (range) }\end{array}$ & $12(3-25)$ & $15(3-25)$ & $15(3-25)$ & $15(4-30)$ & 1.48 & $(1.34-1.63)$ & 1.39 & $(1.26-1.53)$ \\
\hline Time since cessation ${ }^{\ddagger}$, median (range) & $18(1-36)$ & $18(1-36)$ & $16(1-36)$ & $14(1-35)$ & 0.96 & $(0.88-1.05)$ & 0.96 & $(0.88-1.05)$ \\
\hline Smoking duration ${ }^{\S}$, median (range) & $31(4-45)$ & $31(5-45)$ & $31(5-45)$ & $31(5-45)$ & 1.18 & $(1.06-1.32)$ & 1.16 & $(1.04-1.30)$ \\
\hline Alcohol abstainers, \% (N) & $2(146)$ & $2(148)$ & $2(129)$ & $4(187)$ & $2.53 * * *$ & $(1.97-3.26)$ & $2.04 * * *$ & $(1.58-2.63)$ \\
\hline Occasional drinkers, \% (N) & $1(108)$ & $1(97)$ & $2(140)$ & $3(140)$ & $2.11 * * *$ & $(1.57-2.82)$ & $1.71 * * *$ & $(1.28-2.29)$ \\
\hline Alcohol $^{+\dagger \dagger}$, median (range) & $11(1-40)$ & $11(1-41)$ & $10(1-44)$ & $7(1-44)$ & 1.11 & $(1.07-1.14)$ & 1.07 & $(1.03-1.10)$ \\
\hline No sports activity, \% (N) & $37(2,850)$ & $37(2,961)$ & $42(3,165)$ & $52(2,699)$ & $1.88^{\ddagger \ddagger}$ & $(1.66-2.13)$ & $1.53^{\text {势 }}$ & $(1.35-1.73)$ \\
\hline Duration of sports activity, median (range) & $2(0.5-6.5)$ & $1.5(0.5-6)$ & $1.5(0.5-5.5)$ & $1.5(0.5-5)$ & $1.03^{\S \S}$ & $(1.00-1.07)$ & $1.03^{\S \S \$ \ddagger}$ & $(1.00-1.07)$ \\
\hline$<8$ years of school, \% $(\mathrm{N})$ & $24(1,856)$ & $29(2,256)$ & $34(2,550)$ & $41(2,132)$ & 1.00 & - & 1.00 & - \\
\hline $8-10$ years of school, $\%(\mathrm{~N})$ & $52(3,949)$ & $52(4,083)$ & $50(3,805)$ & $47(2,439)$ & 0.81 & $(0.72-0.90)$ & 0.92 & $(0.82-1.04)$ \\
\hline$>10$ years of school, $\%(\mathrm{~N})$ & $24(1,836)$ & $20(1,568)$ & $16(1,214)$ & $13(668)$ & 0.66 & $(0.56-0.78)$ & 0.82 & $(0.69-0.97)$ \\
\hline
\end{tabular}

MMR = Mortality rate ratio.

*Adjusted for covariates related to the same lifestyle aspect.

${ }^{\dagger}$ Adjusted for all other covariates.

${ }^{\ddagger}$ Estimated MMR for smokers with 25 years smoking duration and currently smoking $20 \mathrm{~g}$ /day relative to never smokers.

${ }^{8}$ Smokers who quitted smoking within the last year after 25 years of smoking relative to never smokers. One man who began to smoke and quitted smoking within the last year before recruitment into the study was classifies as a never smoker.

**Estimated MRR for past smokers quitting smoking 15 years ago after 25 years of smoking relative to never smokers.

${ }^{\dagger}$ Median (range) in g/day among current smokers only. MRR per doubling in current tobacco consumption.

Median (range) in years among past smokers only. MRR per doubling of time since cessation.

${ }^{\$ \S}$ Median (range) in years among ever smokers. MRR per doubling of smoking duration.

****Estimated MRR relative to drinkers who drink $10 \mathrm{~g}$ alcohol/day.

${ }^{\dagger}$ Median (range) in grams for average daily alcohol intake among drinkers. MRR per $10 \mathrm{~g}$ increase in average daily alcohol intake.

\#+No sports activity relative to $1 \mathrm{~h}$ of sport activity either summer or winter.

${ }^{\$ \$}$ Median (range) in h/week of duration of sports activity among active. MRR per h/week of sports activity. 
Table 2. Mortality rate ratios (MRR) per $10 \%$ difference of waist circumference and BMI, mutually adjusted, including and omitting the lifestyle aspects one at a time

\begin{tabular}{|c|c|c|c|c|c|c|c|c|}
\hline & \multicolumn{2}{|c|}{ Waist circumference } & \multicolumn{6}{|c|}{$\mathrm{BMI}, \mathrm{kg} / \mathrm{m}^{2 *}$} \\
\hline & \multirow[b]{2}{*}{ MRR } & \multirow[b]{2}{*}{$95 \%$ CI } & \multicolumn{2}{|c|}{ under 25} & \multicolumn{2}{|l|}{$25-30$} & \multicolumn{2}{|c|}{ over 30} \\
\hline & & & MRR & $95 \% \mathrm{CI}$ & MRR & $95 \% \mathrm{CI}$ & MRR & $95 \%$ CI \\
\hline \multicolumn{9}{|l|}{ Men } \\
\hline Without lifestyle covariates $^{\dagger}$ & 1.55 & $1.43-1.67$ & 0.55 & $0.50-0.60$ & 0.91 & $0.83-1.00$ & 0.92 & $0.83-1.02$ \\
\hline Smoking habits adjusted & 1.41 & $1.30-1.53$ & 0.63 & $0.57-0.69$ & 0.97 & $0.89-1.07$ & 0.99 & $0.90-1.09$ \\
\hline Alcohol intake adjusted & 1.46 & $1.35-1.58$ & 0.58 & $0.53-0.63$ & 0.93 & $0.85-1.02$ & 0.95 & $0.86-1.04$ \\
\hline Sports activity adjusted & 1.48 & $1.37-1.61$ & 0.58 & $0.53-0.63$ & 0.90 & $0.82-0.99$ & 0.93 & $0.84-1.02$ \\
\hline Years of schooling adjusted & 1.56 & $1.44-1.69$ & 0.54 & $0.50-0.59$ & 0.88 & $0.81-0.97$ & 0.90 & $0.82-1.00$ \\
\hline Full model ${ }^{\ddagger}$ & 1.34 & $1.24-1.45$ & 0.66 & $0.61-0.72$ & 0.97 & $0.89-1.07$ & 1.00 & $0.91-1.11$ \\
\hline Omitting smoking habits & 1.42 & $1.31-1.54$ & 0.60 & $0.55-0.65$ & 0.90 & $0.82-0.99$ & 0.94 & $0.85-1.03$ \\
\hline Omitting alcohol intake & 1.39 & $1.28-1.50$ & 0.64 & $0.59-0.70$ & 0.95 & $0.87-1.05$ & 0.98 & $0.89-1.08$ \\
\hline Omitting sports & 1.36 & $1.26-1.48$ & 0.65 & $0.59-0.71$ & 0.97 & $0.89-1.07$ & 1.00 & $0.90-1.10$ \\
\hline Omitting years of schooling & 1.33 & $1.23-1.44$ & 0.67 & $0.61-0.73$ & 0.99 & $0.90-1.08$ & 1.01 & $0.92-1.12$ \\
\hline \multicolumn{9}{|l|}{ Women } \\
\hline Without lifestyle covariates $^{\dagger}$ & 1.42 & $1.32-1.52$ & 0.63 & $0.58-0.68$ & 0.87 & $0.78-0.97$ & 0.89 & $0.80-0.99$ \\
\hline Smoking habits adjusted & 1.28 & $1.19-1.38$ & 0.71 & $0.65-0.77$ & 0.94 & $0.84-1.05$ & 0.97 & $0.87-1.08$ \\
\hline Alcohol intake adjusted & 1.38 & $1.28-1.48$ & 0.64 & $0.59-0.70$ & 0.90 & $0.80-1.01$ & 0.89 & $0.80-0.99$ \\
\hline Sports activity adjusted & 1.39 & $1.29-1.50$ & 0.65 & $0.60-0.70$ & 0.85 & $0.75-0.95$ & 0.88 & $0.79-0.98$ \\
\hline Years of schooling adjusted & 1.42 & $1.32-1.52$ & 0.62 & $0.57-0.67$ & 0.85 & $0.76-0.95$ & 0.88 & $0.79-0.99$ \\
\hline Full model ${ }^{\ddagger}$ & 1.25 & $1.16-1.35$ & 0.73 & $0.67-0.79$ & 0.93 & $0.83-1.04$ & 0.95 & $0.85-1.06$ \\
\hline Omitting smoking habits & 1.35 & $1.26-1.46$ & 0.66 & $0.61-0.71$ & 0.86 & $0.77-0.97$ & 0.89 & $0.79-0.99$ \\
\hline Omitting alcohol intake & 1.28 & $1.18-1.37$ & 0.71 & $0.66-0.77$ & 0.91 & $0.81-1.02$ & 0.95 & $0.86-1.06$ \\
\hline Omitting sports & 1.26 & $1.17-1.36$ & 0.72 & $0.66-0.78$ & 0.95 & $0.84-1.06$ & 0.96 & $0.86-1.07$ \\
\hline Omitting years of schooling & 1.25 & $1.16-1.35$ & 0.73 & $0.67-0.79$ & 0.93 & $0.83-1.05$ & 0.95 & $0.86-1.06$ \\
\hline
\end{tabular}

*MRR per $10 \%$ difference within the specific BMI interval.

${ }^{\dagger}$ Waist circumference and BMI simultaneously included in the model.

${ }^{\ddagger}$ Waist circumference, BMI and all covariates simultaneously included in the model.

We evaluated the potential of each of the lifestyle aspects as effect modifier for the association between the body size measurements and allcause mortality by estimating separate MRR for the body size measurements for each of the different levels of the lifestyle factors. The statistical significance was evaluated by testing for each body size measure whether the MRR for the specific body size measure could be assumed to be the same for all the levels of the lifestyle factor, while still allowing the MRR for the other body size measure in the model to depend on the level of the lifestyle factor.

All significance tests and confidence intervals (CI) at the 95\% level were based on Wald's test statistic for the corresponding regression parameters in the Cox regression models, i.e., on the log-scale for the rate ratios. We used the SAS-PHREG procedure (SAS Institute, version 8 for Unix, Cary, NC, USA).

\section{Results}

Between inclusion into the study and December 31, 2005, a total of 3,604 deaths (2,223 men and 1,381 women) occurred in the cohort after exclusion of participants for whom varia- bles of interest were missing. During follow-up 282 participants $(0.5 \%)$ emigrated. The follow-up of vital status for the remaining was complete, except for 4 participants who were impossible to trace in Denmark (true disappearances). The median length of the follow-up period was 9.7 years (1 and 99 percentiles: 2.4 and 11.6 years for the total cohort, and 8.6 and 11.6 years for the survivors).

The distribution of the lifestyle aspects according to waist quartiles among the deceased are shown (table 1). The last two columns in the table show estimated MRR for each lifestyle aspect: crude, i.e. including none of the other lifestyle aspects, and mutually adjusted, i.e. including all other lifestyle covariates. All associations are in the expected direction.

The associations between waist circumference and BMI, mutually adjusted, and all-cause mortality with and without adjustment for lifestyle aspects are shown in table 2. For men, the adjustment for smoking affected the estimated associations with waist circumference and BMI; in some cases, the MRR estimate in models not including smoking was out- 
Table 3. Mortality rate ratios (MRR) for waist and hip circumferences, mutually adjusted, including and omitting the lifestyle covariates one at a time

\begin{tabular}{|c|c|c|c|c|c|c|c|c|}
\hline & \multicolumn{4}{|l|}{ Men } & \multicolumn{4}{|c|}{ Women } \\
\hline & \multicolumn{2}{|c|}{$\begin{array}{l}\text { waist circumference per } \\
10 \% \text { higher }\end{array}$} & \multicolumn{2}{|c|}{$\begin{array}{l}\text { hip circumference per } \\
10 \% \text { lower }\end{array}$} & \multicolumn{2}{|c|}{$\begin{array}{l}\text { waist circumference } \\
\text { per } 10 \% \text { higher }\end{array}$} & \multicolumn{2}{|c|}{$\begin{array}{l}\text { hip circumference per } \\
10 \% \text { lower }\end{array}$} \\
\hline & MRR & $95 \% \mathrm{CI}$ & MRR & $95 \% \mathrm{CI}$ & MRR & $95 \% \mathrm{CI}$ & MRR & $95 \% \mathrm{CI}$ \\
\hline Without lifestyle covariates" & 1.61 & $1.51-1.71$ & 1.81 & $1.64-1.98$ & 1.32 & $1.25-1.40$ & 1.58 & $1.44-1.72$ \\
\hline Smoking habits adjusted & 1.48 & $1.39-1.57$ & 1.52 & $1.38-1.67$ & 1.23 & $1.16-1.30$ & 1.34 & $1.23-1.47$ \\
\hline Alcohol intake adjusted & 1.52 & $1.43-1.62$ & 1.69 & $1.54-1.86$ & 1.30 & $1.23-1.37$ & 1.53 & $1.40-1.67$ \\
\hline Sports activity adjusted & 1.52 & $1.42-1.61$ & 1.71 & $1.56-1.88$ & 1.29 & $1.22-1.37$ & 1.56 & $1.43-1.71$ \\
\hline Years of schooling adjusted & 1.56 & $1.47-1.67$ & 1.76 & $1.60-1.93$ & 1.31 & $1.24-1.38$ & 1.57 & $1.44-1.71$ \\
\hline Full model $^{\dagger}$ & 1.38 & $1.29-1.47$ & 1.41 & $1.28-1.55$ & 1.19 & $1.13-1.27$ & 1.32 & $1.21-1.45$ \\
\hline Omitting smoking habits & 1.42 & $1.33-1.51$ & 1.58 & $1.44-1.74$ & 1.25 & $1.19-1.33$ & 1.50 & $1.38-1.64$ \\
\hline Omitting alcohol intake & 1.42 & $1.33-1.52$ & 1.47 & $1.33-1.62$ & 1.21 & $1.14-1.28$ & 1.35 & $1.23-1.48$ \\
\hline Omitting sports & 1.41 & $1.32-1.50$ & 1.43 & $1.30-1.58$ & 1.20 & $1.14-1.28$ & 1.32 & $1.20-1.44$ \\
\hline Omitting years of schooling & 1.39 & $1.31-1.48$ & 1.42 & $1.29-1.57$ & 1.20 & $1.13-1.27$ & 1.32 & $1.21-1.45$ \\
\hline
\end{tabular}

* Waist and hip circumferences simultaneously included in the model.

$\dagger$ Waist and hip circumferences and all lifestyle covariates simultaneously included in the model.

side the confidence limits seen in the corresponding analysis including the smoking covariates (waist circumference, per $10 \%$ difference, without covariates MRR $=1.55$ while adjusted for smoking MRR 1.41, 95\% CI 1.30-1.53; BMI below $25 \mathrm{~kg} / \mathrm{m}^{2}$, per $10 \%$ difference, without covariates MRR 0.55 while adjusted for smoking MRR $0.63,95 \%$ CI $0.57-0.69$; and full model MRR 0.66, 95\% CI 0.61-0.72, while MRR was 0.60 when smoking was omitted). The adjustment for alcohol intake had a modest effect on the estimated association with waist circumference, whereas adjustment for education had no effect on the estimated MRR associated with waist circumference and BMI. The adjustment for sport activity showed only modest effect on the MRR estimate for waist circumference when sport activity was included as the only covariate, and no effect when the other lifestyle aspects were included. For women, the adjustment for smoking affected the MRR estimates for the body size measurements while the adjustment for alcohol intake, although significant as a risk factor, showed only modest effect on the MRR estimates, and none of the other lifestyle aspects showed any sign of confounding.

The association between all-cause mortality and the waist (per 10\% larger) and hip (per 10\% smaller) circumferences is shown in table 3 , mutually adjusted, with and without adjustment for lifestyle covariates. For both men and women, the adjustment for smoking showed the strongest effect on the MRR estimates for both circumferences; the MRR estimate in models not including smoking was generally outside the confidence limits seen in the corresponding analysis including the smoking covariates except for the MRR for the waist circumference in the comparison with the full model. For the men, adjustment for alcohol intake and not performing sports had a moderate influence on estimates. Adjusting for sports activity was most pronounced for the MRR associated with the waist circumference, but made no difference when combined with the other lifestyle aspects. For women, there was little difference between the smoking-adjusted model (waist MRR 1.23, 95\% CI 1.16-1.30; hip MRR 1.34, 95\% CI $1.23-$ 1.47 ) and the fully adjusted model (waist MRR $1.20,95 \%$ CI 1.13-1.27; hip MRR 1.32, 95\% CI 1.21-1.45), showing that the other lifestyle aspects had very little confounding potential.

If the waist-to-hip ratio is the adequate measure of the association between mortality and the two circumferences (table 3), then the two estimates should be equal; thus the waist-to-hip-ratio seemed adequate in men (in the full model, per $10 \%$ higher waist: MRR $1.38,95 \%$ CI $1.29-1.47$; per $10 \%$ lower hip: MRR 1.41, 95\% CI 1.28-1.55), but not in women. (per 10\% higher waist: MRR 1.19, 95\% CI 1.13-1.32; per $10 \%$ lower hip: MRR 1.32, 95\% CI:1.21-1.45).

Table 4 shows the estimated MRR for the body size measurements for different levels of each of the four selected lifestyle aspects; smoking habits, alcohol intake, physical activity and years of schooling. For all levels of the lifestyle factors, the same general pattern as in the overall analyses was seen, that is, the mortality increased with higher waist circumference, decreased with higher BMI below $25 \mathrm{~kg} / \mathrm{m}^{2}$ and increased with lower hip circumference. Statistically significant signs of some degree of effect modification were seen a few. However, in all cases the statistical significance was caused by a deviation in the body size measurement MRR for the intermediate level of the lifestyle factor to the two extreme levels of the lifestyle factor. 


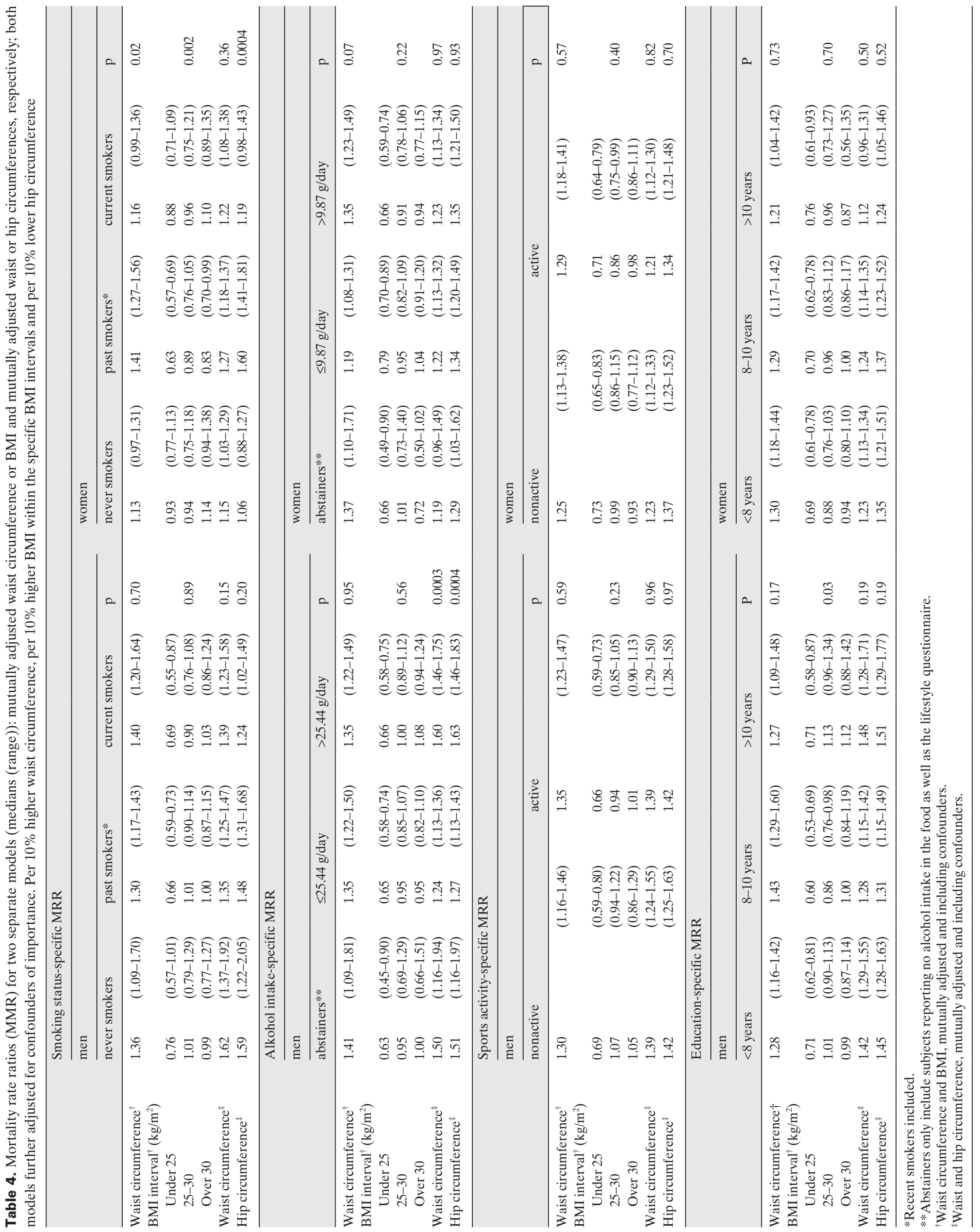




\section{Discussion}

The influence of the body size measurements on mortality remained strong after adjustment for the lifestyle aspects: smoking habits, alcohol intake, sports activity and education. The general pattern for both sexes was a steadily increasing mortality rate with higher waist circumference for a given BMI or hip circumference and a steadily decreasing mortality rate with higher hip circumference for a given waist circumference, whereas the association between the mortality rate and BMI for a given waist circumference was limited to individuals with low BMI (BMI below $25 \mathrm{~kg} / \mathrm{m}^{2}$ ) where the mortality was strongly increasing with lower BMI. The same pattern was seen for all categories for the lifestyle variables, indicating that the potential effect modification by the lifestyle factors was not an important issue, although a few statistical significances were seen where the estimated associations between mortality and the body size measurements for the two extreme levels of the lifestyle factor were similar while the estimated associations for the intermediate level deviated to some degree.

Adjustment for smoking habits attenuated the associations in both men and women while adjustment for alcohol intake and sport activity attenuated association for men only. Furthermore, adjustment for the different lifestyle aspects affected the association with waist circumference and hip circumference differently, supporting the use of the separate measurements of waist and hip circumference instead of the waist-to-hip ratio.

The arguments for selecting the four lifestyle aspects were as follows: Manson and coworkers [10,11] recommended including smoking variables in models studying the relation between BMI and mortality because a spuriously high mortality would otherwise be produced in the low range of BMI. However, the U-shape remained in many studies despite inclusion of smoking covariates $[1,12]$ possibly due to BMI representing separate and opposite associations of fat mass and fat free mass with mortality [13-17]. Physical activity cannot be ruled out as a part of the causal pathway between obesity and mortality as a cause and/or a consequence of obesity; but since physically inactive individuals seem to have a higher risk of mortality independently of level of adiposity and excess adiposity seem to be associated with higher mortality independently of physical activity [4], physical activity and adiposity are not just two aspects of a single cause, so confounding could still be an issue. Alcohol seems to be independently associated with all-cause mortality in a U- or J-shaped relation [3]. The descending leg of the association may result from a decreased risk of cardiovascular disease among those with light to moderate alcohol intake [3] or may reflect a higher mortality among abstainers, a group that includes people who refrain from alcohol intake because of disease or earlier alcohol abuse [18]. Education was selected as a proxy for social position, which is inversely associated with all-cause mortality [5].
In this study, we investigated the separate effects of adjustment for different lifestyle aspects on associations between body size measurements and mortality. Earlier papers most often show crude and multivariate adjusted associations, but not the effect of including only a single lifestyle factor, and this makes it impossible to evaluate the importance of the adjustment for each specific lifestyle factor. Therefore, we elaborated on attenuations of associations between mortality and the measurements of waist circumference, BMI and hip circumference by including and excluding lifestyle aspects one at a time. Furthermore, we investigated whether the lifestyle aspects modified the associations between mortality and the body size measurements by presenting these associations estimated separately for different levels of the lifestyle factors.

We have investigated the potential confounding and effect modification from smoking habits in other papers [1, 2]. In analyses of waist circumference and BMI, mutually adjusted, with all-cause mortality as the outcome, we found that adjustment for smoking habits attenuated the associations [1]. In the adjustment for smoking habits, we considered smoking status (current, past, and never use of tobacco), current tobacco consumption in grams, smoking duration and time since cessation [1]. Some reviews have suggested that smoking modifies the effect of obesity $[10,11]$; however, we found similar association for never smokers and current smokers for both men and women, while past smokers among women showed a stronger association. The first study to suggest effect modification by smoking on the association between adiposity and mortality was the Framingham Heart Study [19], but recent re-analyses including longer follow-up could not confirm the earlier findings [20]. Our findings suggest that smoking is an important confounder in men and women, which attenuates but far from eliminate the association of mortality with the body size measurements, but there was no sign of effect modification by current smoking.

We found a significant adverse association with increasing alcohol intake in general for both sexes, but also an increased mortality among occasional drinkers and those who claimed to be abstainers. This is in agreement with a British study considering the association between baseline alcohol intake among men as well as variation in alcohol intake over time with all-cause mortality [21]. They found a U-shaped relationship with baseline alcohol intake but a linear relationship when variation in alcohol intake was considered, except for occasional drinkers who had a slightly increased mortality risk compared to alcohol abstainers [21]. Also overweight individuals may benefit from a low to moderate intake of alcohol reducing their mortality risk [22]. Although the alcohol was an important risk factor for both sexes in the present study, adjusting for alcohol intake had only modest effect on the estimated associations between mortality and the body size measurements for men and no effect for women. In the present study, we saw indications of a less strong increase of 
the mortality rate with higher waist-to-hip ratio among men with intermediate daily alcohol intake.

An extensive review of leisure-time physical activity (data obtained by questionnaires) and all-cause mortality summarized the relationships from published studies using metaanalysis and concluded that both physical inactivity and adiposity are important determinants of mortality risk from all causes [4]. Physical inactivity is associated with a higher risk of mortality independently of the adiposity level, and excess adiposity is associated with a higher risk of mortality independently of physical activity level [4]. Two recently published follow-up studies came to similar conclusions in separate analyses of physical inactivity and all-cause mortality in men aged 35-65 years [23] and women aged 30-55 years [24]. Our findings support the conclusion from these studies; lack of sports activity was a strong indicator for increased mortality risk for both sexes (table 1). A recent study considering cardiorespiratory fitness showed that the association between waist circumference and mortality disappeared after adjustment for fitness [25]. In the present study, adjustment for sports activity made very little difference for the estimated effects of the body size measurements in spite of the strong association seen between sports activity and mortality in this study.

Education (years of schooling) was used as a proxy variable for socioeconomic status known to influence both mortality [26] and body size [27] independently. The crude analyses of education and mortality showed a tendency to an inverse relationship, so individuals with less than 8 years of schooling had a higher mortality, but the associations became insignificant when adjusted for the other lifestyle aspects. In general, the participants in the study have higher socioeconomic position than non-participants [6]. Although years of schooling showed no confounding in the present study, this cannot be generalized to an assumption of no confounding potential of other indicators of social inequalities.

Our study had a median follow-up time of 9.7 years, and 3,604 deaths occurred during the study. The large number of events allows for inclusion of several covariates in the analyses as well as investigations of signs of effect modifications, which was not possible in earlier papers. Detailed information about covariates was obtained from self-administered questionnaires, supplemented by a personal interview in the study clinic if answers were unclear [6]. Laboratory technicians obtained all body size measurements, so the analyses did not depend on self-reported measurements which are known to be biased [18]. Only one third (35\%) of the invited persons participated and according to age- and sex-specific death rates of the general Danish population, only one half and one third of the expected numbers of deaths among men and women, respectively, had occurred at the end of 1999 [28]. This is probably due to an underrepresentation of lower socioeconomic groups [6]. Also the exclusion of subjects with previously diagnosed cancer and/or an overall better level of health among the participants lead to a 'healthy participant effect'. There seems, however, to be no reason to assume that the observed pattern for the attenuations of associations between body size measurements and mortality cannot be extrapolated to non-participants. The study was limited to the age range of 50-70 years, and the extrapolation beyond this range may not be valid. Epidemiological investigations using self-reported questionnaire data has limitations due to the risk of differential and non-differential misclassifications leading to information bias. However, in view of the prospective nature of the study where the outcome is unknown to the participants at the baseline reporting, differential misclassification is unlikely. Furthermore, although the estimated effect of a confounder may be biased, non-differential overreporting or underreporting will not affect the degree to which confounding can be controlled for as long as the ranking of the subjects reflects reality. Waist-to-height ratio is not included in this paper, but is an interesting perspective in future research.

In summary, these investigations found evidence of smoking habits to be the most important confounder of the investigated associations between the body size measurements and mortality for both sexes. Alcohol intake and sports activity were weak confounders among men, but not among women. The associations between the body size measurements and mortality remained strong and significant after adjustment for the lifestyle aspects, and the same pattern for the associations was seen for all levels for the different lifestyle factors. Our study confirm that adiposity related to waist circumference (apple shape) is hazardous, while adiposity related to the hips and buttocks (pear shape) seems to be protective, and that very low BMI is associated with a higher mortality rate in both men and women also after adjusting for lifestyle aspects.

\section{Acknowledgments}

The authors thank all participants in the study. We also thank secretary Jytte Fogh Larsen and programmer Katja Boll for collection and preparation of the dataset. Dr. Bigaard had full access to all of the data in the study and takes responsibility for the integrity of the data and the accuracy of the data analyses. The study concept and design was done by Bigaard, Thomsen, Tjønneland, Overvad and Sørensen. Tjønneland and Overvad were responsible for the acquisition of data. Bigaard, Thomsen and Christensen were responsible for the analysis and interpretation of data. Bigaard drafted the article. Bigaard, Thomsen, Tjønneland, Overvad and Sørensen were responsible for the critical revision of the article for important intellectual content. Christensen and Thomsen were responsible for the statistical analyses. Tjønneland, Overvad and Sørensen obtained funding.

\section{Disclosure}

The authors declare no conflict of interest. 


\section{References}

1 Bigaard J, Tjønneland A, Thomsen BL, Overvad K, Heitmann BL, Sørensen TI: Waist circumference, BMI, smoking, and mortality in middle-aged men and women. Obes Res 2003;11:895-903.

${ }_{2}$ Bigaard J, Frederiksen K, Tjønneland A et al: Waist and hip circumferences and all-cause mortality: usefulness of the waist-to-hip ratio? Int J Obes Relat Metab Disord 2004;28:741-747.

3 Grønbæk M: Alcohol, type of alcohol, and allcause and coronary heart disease mortality. Ann N Y Acad Sci 2002;957:16-20.

4 Katzmarzyk PT, Janssen I, Ardern CI: Physical inactivity, excess adiposity and premature mortality. Obes Rev 2003;4:257-290.

5 Mustard CA, Etches J: Gender differences in socioeconomic inequality in mortality. J Epidemiol Community Health 2003;57:974-980.

6 Tjønneland A, Olsen A, Boll K, Stripp C, Christensen J, Engholm G, Overvad K: Study design, exposure variables, and socioeconomic determinants of participation in Diet, Cancer and Health a population-based prospective cohort study of 57,053 men and women in Denmark. Scand J Public Health 2007;35:432-441.

$\checkmark 7$ Korn EL, Graubard BI, Midthune D: Time-toevent analysis of longitudinal follow-up of a survey: choice of the time-scale. Am J Epidemiol 1997;145: $72-80$.

8 Greenland S: Dose-response and trend analysis in epidemiology: alternatives to categorical analysis. Epidemiology 1995;6:356-365.

9 WHO: Obesity: Preventing and Managing the Global Epidemic. Report of a WHO Consultation. Geneva, World Health Organization, 2000.

10 Manson JE, Stampfer MJ, Hennekens CH, Willett WC: Body weight and longevity. A reassessment JAMA 1987;257:353-358.

11 Solomon CG, Manson JE: Obesity and mortality: a review of the epidemiologic data. Am J Clin Nutr 1997;66(4 suppl):1044S-1050S.

12 Pischon T, Boeing H, Hoffmann K et al: General and abdominal adiposity and risk of death in $\mathrm{Eu}$ rope. N Engl J Med 2008;359:2105-2120.

-13 Baumgartner RN, Heymsfield SB, Roche AF: Human body composition and the epidemiology of chronic disease. Obes Res 1995;3:73-95.

14 Heitmann BL, Erikson H, Ellsinger BM, Mikkelsen KL, Larsson B: Mortality associated with body fat, fat free mass and body mass index among 60-year-old Swedish men - a 22 year follow-up. The study of men born in 1913. Int J Obes Relat Metab Disord 2000;24:33-37.

15 Bigaard J, Frederiksen K, Tjønneland A, Thomsen BL, Overvad K, Heitmann BL, Sørensen TIA: Body fat and fat-free mass and all-cause mortality. Obes Res 2004;12:1042-1049.

16 Janssen I, Katzmarzyk PT, Ross R: Body mass index is inversely related to mortality in older people after adjustment for waist circumference. J Am Geriatr Soc 2005;53:2112-2118.

17 Wannamethee SG, Shaper AG, Lennon L, Whincup PH: Decreased muscle mass and increased central adiposity are independently related to mortality in older men. Am J Clin Nutr 2007;86:1339-1346.

18 Bigaard J, Spanggaard I, Thomsen BL, Overvad K, Tjønneland A: Self-reported and technician-measured waist circumferences differ in middle-aged men and women. J Nutr 2005;135:2263-2270.

19 Garrison RJ, Feinleib M, Castelli WP, McNamara PM: Cigarette smoking as a confounder of the relationship between relative weight and long-term mortality. The Framingham Heart Study. JAMA 1983;249:2199-2203.
20 Sempos CT, Durazo-Arvizu R, McGee DL, Cooper RS, Prewitt TE: The influence of cigarette smoking on the association between body weight and mortality. The Framingham Heart Study revisited. Ann Epidemiol 1998;8:289-300.

21 Emberson JR, Shaper AG, Wannamethee SG, Morris RW, Whincup PH: Alcohol intake in middle age and risk of cardiovascular disease and mortality: accounting for intake variation over time. Am J Epidemiol 2005;161:856-863.

22 Dixon JB, Dixon ME, O'Brien PE. Alcohol consumption in the severely obese: relationship with the metabolic syndrome. Obes Res 2002;10:245-252.

$23 \mathrm{Hu}$ FB, Willett WC, Li T, Stampfer MJ, Colditz GA, Manson JE: Adiposity as compared with physical activity in predicting mortality among women. N Engl J Med 2004;351:2694-2703.

$24 \mathrm{Hu}$ G, Tuomilehto J, Silventoinen K, Barengo NC, Peltonen M, Jousilahti P: The effects of physical activity and body mass index on cardiovascular, cancer and all-cause mortality among 47212 middle-aged Finnish men and women. Int J Obes (Lond) 2005;29:894-902.

25 Sui X, LaMonte MJ, Laditka JN, Hardin JW, Chase N, Hooker SP, Blair SN: Cardiorespiratory fitness and adiposity as mortality predictors in older adults. JAMA 2007;298:2507-2516.

26 Osler M, Prescott E: Educational level as a contextual and proximate determinant of all cause mortality in Danish adults. J Epidemiol Community Health 2003;57:266-269.

27 Molarius A, Seidell JC, Sans S, Tuomilehto J, Kuulasmaa K: Educational level, relative body weight, and changes in their association over 10 years: an international perspective from the WHO MONICA Project. Am J Public Health 2000;90:1260-1268.

28 Tjønneland A, Overvad OK. Diet, cancer and health--a population study and establishment of a biological bank in Denmark (in Danish). Ugeskr Laeger 2000;162:350-354. 